\title{
A Case of CIED-Associated Endocarditis and Septic Emboli Requiring Lead Extraction, AngioVac Suction, and Pulmonary Endarterectomy
}

\author{
Erik A. Green ${ }^{1}$, Travis Pollema ${ }^{2}$, Victor Pretorius ${ }^{2}$ \\ 1. Surgery, Tulane University School of Medicine, New Orleans, USA 2. Cardiothoracic Surgery, University of California \\ San Diego School of Medicine, La Jolla, USA
}

Corresponding author: Erik A. Green, eagreen08@gmail.com

\begin{abstract}
Cardiac Implantable Electronic Devices (CIED)-associated infective endocarditis complicated by septic emboli and acute on chronic pulmonary hypertension is rare. We present a case where pulmonary thromboendarterectomy was required for treatment. A 55 year-old man with a history of myocardial infarction and ischemic cardiomyopathy status-post ICD placement 8 years prior presented with bacteremia, infected ICD, and tricuspid valve vegetation. He underwent CIED extraction along with the use of the AngioVac suction device to remove right ventricular and atrial vegetations. However the patient had persistent valvular vegetation and bilateral sub-massive pulmonary emboli. Pulmonary angiography showed filling defects in the lobar and segmental arteries. Percutaneous attempts at embolectomy were unsuccessful and he therefore underwent a pulmonary endarterectomy surgery (PTE).
\end{abstract}

This case of CIED- associated endocarditis demonstrates the importance of early aggressive treatment of such infections. Guidelines recommend compete CIED system removal when there is associated infection. The AngioVac is a novel system for removal of right-sided vegetations and thrombi; however, complications such as distal embolization can occur. PTE surgery for septic emboli is rare. However, cases of such treatment as is presented here can be successful and may be necessary should percutaneous methods fail.

Review began 10/15/2020 Review ended 11/08/2020 Published 11/20/2020

(c) Copyright 2020

Green et al. This is an open access article distributed under the terms of the Creative Commons Attribution License CC-BY 4.0., which permits unrestricted use, distribution, and reproduction in any medium, provided the original author and source are credited.
Categories: Cardiac/Thoracic/Vascular Surgery, Cardiology

Keywords: device-associated endocarditis, septic emboli, lead extraction, angiovac, pulmonary

thromboendarterectomy

\section{Introduction}

Cardiac Implantable Electronic Devices (CIED)-associated infective endocarditis complicated by septic emboli and acute on chronic pulmonary hypertension is rare. Here we present a case where pulmonary thromboendarterectomy (PTE) was required to successfully treat this uncommon complication.

\section{Case Presentation}

A 55 year-old man with a history of myocardial infarction and ischemic cardiomyopathy status-post ICD placement 8 years prior presented to an outside hospital with bacteremia. His original ICD placement was in 2005, with a Guidant FineLine atrial lead and a Guidant dual coil ICD lead. In 2008, an additional Guidant atrial lead was placed. He was transferred to our tertiary care center after 8 weeks of IV antibiotics and evidence of a large $5 \mathrm{~cm} \times 2 \mathrm{~cm}$, mobile tricuspid valve vegetation associated with the ICD lead (Figure 1) seen on trans-Esophageal Echocardiogram (TEE). Blood cultures were positive for Staphylococcus hominis. 


\section{Cureus}

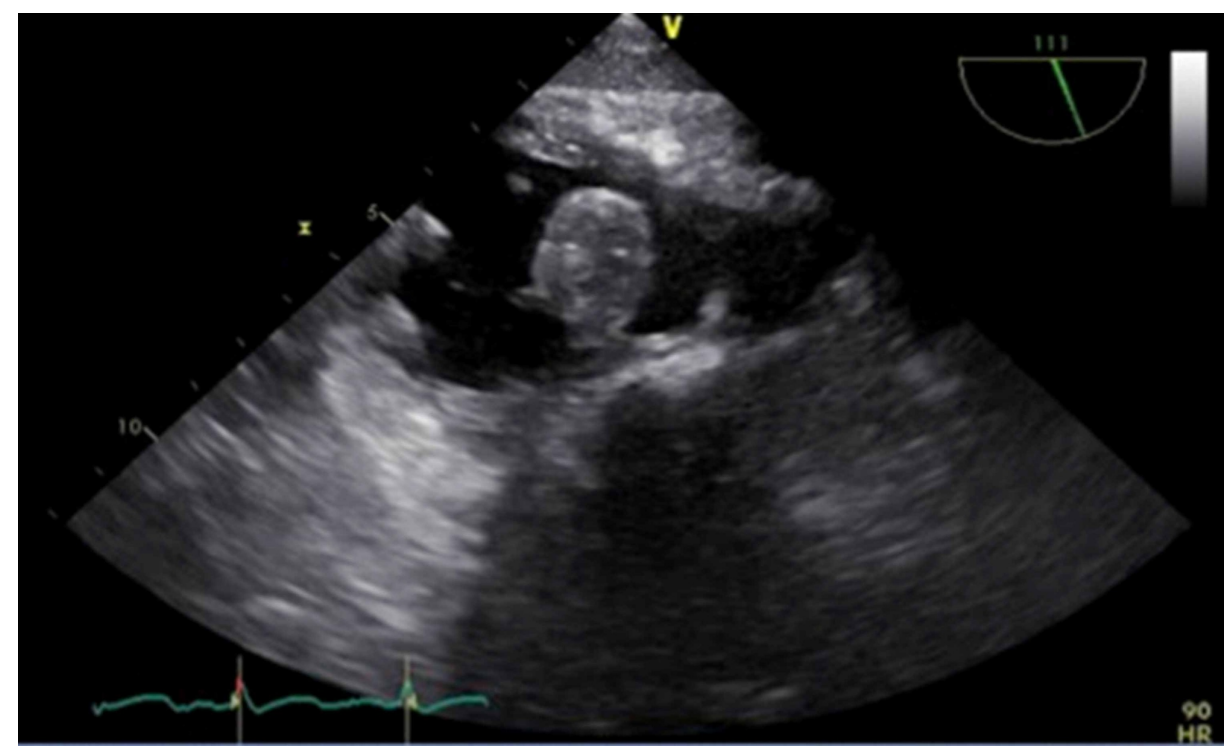

FIGURE 1: TEE imaging showing large mobile tricuspid valve vegetation associated with the CIED lead

The patient was taken to the hybrid OR, placed under general anesthesia, and underwent pulse generator and lead extraction, along with AngioVac removal of RV and RA vegetations (Figure 2). Significant purulent fluid was found in the device pocket and tracking along the leads. The RA FineLine lead was extracted with a $14 \mathrm{~F}$ laser sheath. However, adhesions made extraction difficult and the lead tip was fractured and was subsequently removed using a femoral approach with a snare. The AngioVac system was then used to remove the large vegetation via right internal jugular and right femoral vein cannulation. The patient was heparinized to an ACT $>250$. The AngioVac cannula was advanced into the RA, and $1.5-2 \mathrm{~L} / \mathrm{min}$ flow was achieved. The $14 \mathrm{~F}$ laser sheath was used to extract the second atrial lead and a $16 \mathrm{~F}$ laser sheath and a mechanical rotational tool was then required to extract the ICD lead.

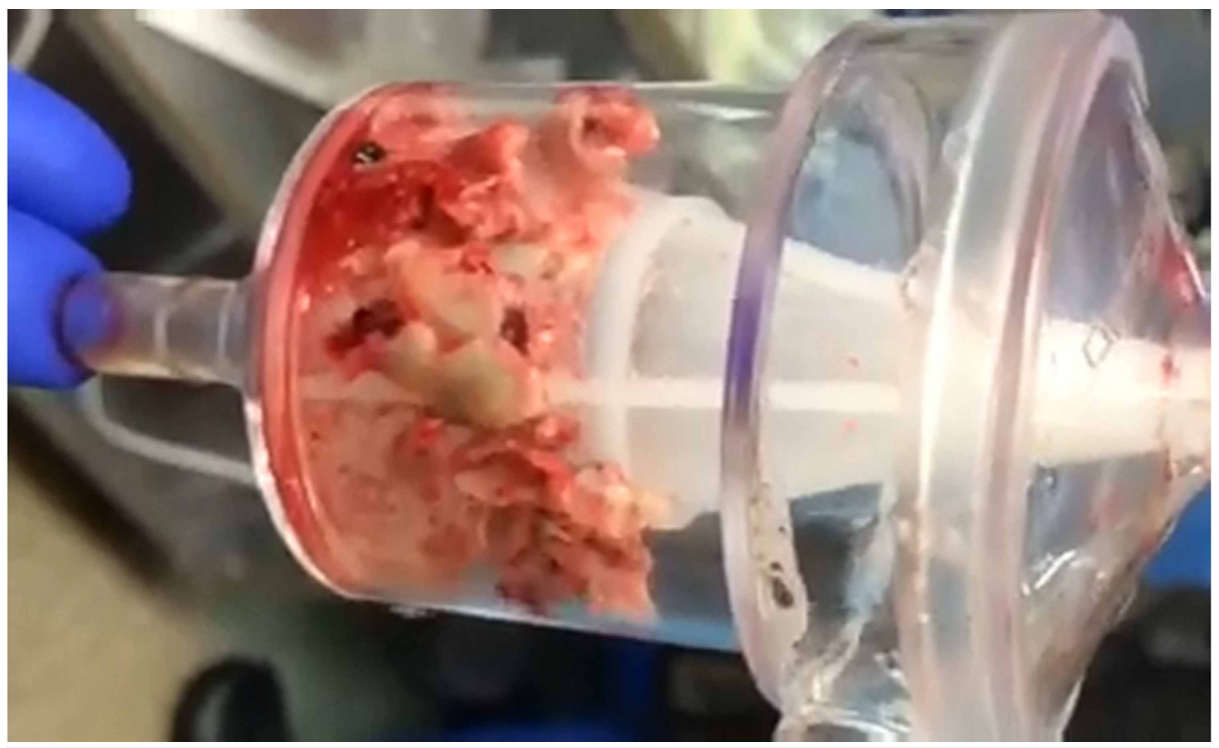

FIGURE 2: RA vegetation and debris post AngioVac suction

Post-procedure the patient remained febrile and with leukocytosis to 23,000. Post-procedure TEE showed persistent valve vegetation and a chest CT scan showed evidence of sub-massive pulmonary emboli. The patient had worsening fevers, chills, pleuritic pain, and dyspnea despite antibiotics. Right heart catheterization revealed normal right heart pressures and borderline pulmonary hypertension with a mean pulmonary artery pressure of $27 \mathrm{mmHg}$. Pulmonary angiography showed proximal filling defects of bilateral lobar and segmental branches. Percutaneous embolectomy was attempted but was unsuccessful as a wire was unable to be passed beyond the thrombus. 


\section{Cureus}

The patient was therefore considered for pulmonary endarterectomy surgery. He underwent bilateral pulmonary endarterectomy under cardiopulmonary bypass and deep hypothermic circulatory arrest (287 minutes of bypass time, 23 minutes of arrest time on right side, 20+18 minutes of arrest on left) (Figure 3). The tricuspid valve and right ventricle vegetations were also removed and the septal leaflet of the tricuspid valve repaired with a Gortex neochord. His post-operative course was uneventful with resolution of symptoms and improved hemodynamics with a Pulmonary Vascular Resistance of 146, down from 316 dyne*sec/cm5. He was discharged on antibiotics and 6 months of anticoagulation. He has done well in follow-up and a new ICD system was later successfully placed at his referring institution.

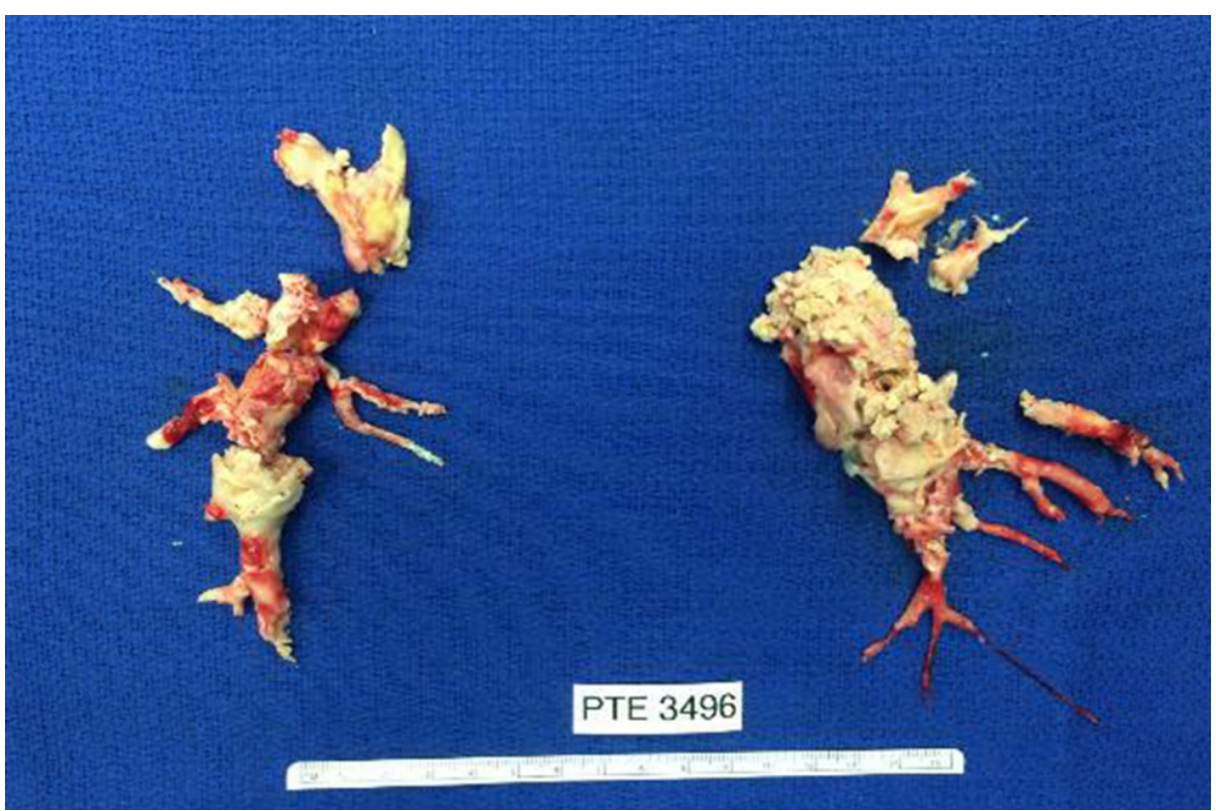

FIGURE 3: Left and Right Pulmonary Endarterectomy specimen

\section{Discussion}

This case of CIED- associated endocarditis demonstrates the importance of early aggressive treatment of such infections and also highlights the importance of strategic lead management.

The 2017 Heart Rhythm Society (HRS) lead management guidelines [1] emphasize the importance of recognizing CIED and lead infection early and that quick implementation of appropriate antibiotic therapy should be initiated. Infection is one of the strongest indications for device removal. The HRS consensus issued a class I recommendation for complete CIED and lead removal within 3 days of infectious diagnosis [1]. Delay of removal, even with appropriate antibiotics, increases the risk of mortality and morbidity, as in this case when the patient came to extraction only after 8 weeks of antibiotic therapy. In CIED infection with associated vegetations, the HRS lead management guidelines similarly suggest device reimplantation after completion of appropriate therapy with 4-6 weeks of antibiotics, depending on the microbiology, and confirmed negative blood cultures [1].

The AngioVac system is a relatively novel method for removal of material from the vasculature using venovenous bypass. Studies have shown it to be an effective method of percutaneous removal of thrombi and right-sided vegetations [2-4]. Reports have also shown it to be useful in management of vegetations associated with pacemaker and defibrillator leads [5-6]. However, as in the patient presented here, cases of incomplete extraction and distal embolization have been reported. This may be more common with chronic or well-organized clots [7-8].

There are a few reports of PTE for septic emboli in the literature [9-10]; however, none associated with a CIED. These few include reports of successful outcomes as well as a report of two cases that were unsuccessful due to inadequate separation between the vessel walls and the embolic masses. In the case presented here, surgery was successful and the patient did well.

\section{Conclusions}

In this case report, we demonstrate a successful treatment of a complicated case of CIED-associated infection and endocarditis. This patient did not undergo early device removal, and despite efforts at minimally invasive techniques of lead and vegetation removal, our patient eventually required an open surgery. This shows the importance of early antibiotic therapy to minimize the development and growth of 
vegetations, little delay in device removal, and close monitoring to determine when to proceed with an open extraction.

\section{Additional Information \\ Disclosures}

Human subjects: Consent was obtained by all participants in this study. Conflicts of interest: In compliance with the ICMJE uniform disclosure form, all authors declare the following: Payment/services info: All authors have declared that no financial support was received from any organization for the submitted work. Financial relationships: All authors have declared that they have no financial relationships at present or within the previous three years with any organizations that might have an interest in the submitted work. Other relationships: All authors have declared that there are no other relationships or activities that could appear to have influenced the submitted work.

\section{References}

1. Kusumoto FM, Schoenfeld MH, Wilkoff BL, et al.: 2017 HRS expert consensus statement on cardiovascular implantable electronic device lead management and extraction. Heart Rhythm. 2017, 14:e503-551. 10.1016/j.hrthm.2017.09.001

2. Worku B, Salemi A, D’Ayala MD, Tranbaugh RF, Girardi LN, Gulkarov IM: The AngioVac device: understanding the failures on the road to success. Innovations (Phila). 2016, 11:430-433. 10.1097/IMI.0000000000000310

3. Thiagaraj AK, Malviya M, Htun WW, Telila T, Lerner SA, Elder MD, Schreiber TL: A novel approach in the management of right-sided endocarditis: percutaneous vegectomy using the AngioVav cannula. Future Cardiol. 2017, 13:211-217. 10.2217/fca-2016-0076

4. George B, Voelkel A, Kotter J, Leventhal A, Gurley J: A novel approach to percutaneous removal of large tricuspid valve vegetations using suction filtration and veno-venous bypass: a single center experience. Catheter Cardiovasc Interv. 2017, 15:1009-1015. 10.1002/ccd.27097

5. Schaerf RHM, Najibi S, Conrad J: Percutaneous vacuum-assisted thrombectomy device used for removal of large vegetations on infected pacemaker and defibrillator leads as an adjunct to lead extraction. J Atr Fibrillation. 2016, 31:1455. 10.4022/jafib.1455

6. Jones BM, Wazni O, Rehm SJ, Shishehbor MH: Fighting fungus with a laser and a hose: management of a giant Candida albicans implantalble cardioberter-defibrillator lead vegetation with simultaneous AngioVac aspiration and laser sheath lead extraction. Catheter Cardiovasc Interv. 2018, 91:318-321. $10.1002 / \mathrm{ccd} .27153$

7. Del Rosario T, Basta M, Agarwal S: AngioVac suction thrombectomy complicated by thrombus fragmentation and distal embolization leading to hemodynamic collapse: a case report. A A Case Rep. 2017, 15:206-209. 10.1213/XAA.0000000000000469

8. Moriarty JM, Al-Hakim R, Bansal A, Park JK: Removal of caval and right atrial thrombi and masses using the AngioVac device: initial operative experience. J Vasc Interv Radiol. 2016, 27:1584-1591. 10.1016/j.jvir.2016.03.045

9. Crosland W, Ahmed M, Booker J, Wille K, Singh S, Winokur T, McGiffin D: Pulmonary endarterectomy for pulmonary hypertension from septic emboli. Ann Thorac Surg. 2015, 99:1814-1816. 10.1016/j.athoracsur.2014.06.109

10. Heiberg J, Ilkjaer LB: Pulmonary endarterectomy after pulmonary infectious embolisms . Interact Cardiovasc Thorac Surg. 2013, 16:556-7. 10.1093/icvts/ivs523 